\title{
The influence of action video game playing on eye movement behaviour during visual search in abstract, in-game and natural scenes
}

\author{
Elham Azizi $^{1} \cdot$ Larry A. Abel $^{1} \cdot$ Matthew J. Stainer $^{2}$
}

Published online: 15 December 2016

(C) The Psychonomic Society, Inc. 2016

\begin{abstract}
Action game playing has been associated with several improvements in visual attention tasks. However, it is not clear how such changes might influence the way we overtly select information from our visual world (i.e. eye movements). We examined whether action-video-game training changed eye movement behaviour in a series of visual search tasks including conjunctive search (relatively abstracted from natural behaviour), game-related search, and more naturalistic scene search. Forty nongamers were trained in either an action first-person shooter game or a card game (control) for 10 hours. As a further control, we recorded eye movements of 20 experienced action gamers on the same tasks. The results did not show any change in duration of fixations or saccade amplitude either from before to after the training or between all nongamers (pretraining) and experienced action gamers. However, we observed a change in search strategy, reflected by a reduction in the vertical distribution of fixations for the game-related search task in the action-game-trained group. This might suggest learning the likely distribution of targets. In other words, game training only skilled participants to search game images for targets important to the game, with no indication of transfer to the more natural scene search. Taken together, these results suggest no modification in overt allocation of attention. Either the skills that can be trained with action gaming are not powerful enough to influence information selection through eye movements, or action-game-
\end{abstract}

Elham Azizi

azizie@unimelb.edu.au

1 Department of Optometry and Vision Sciences, University of Melbourne, Alice Hoy Building, Level 4, 3010 Victoria, Australia

2 School of Psychology, University of Aberdeen, Aberdeen, Scotland learned skills are not used when deciding where to move the eyes.

Keywords Game training · Eye movements · Visual search · Learning $\cdot$ Eye guidance

It has been suggested that playing action video games is associated with improvements in various aspects of visual attention (e.g. Bejjanki et al., 2014; Cain, Landau, \& Shimamura, 2012; Feng, Spence, \& Pratt, 2007; Green \& Bavelier, 2003, 2006; Hubert-Wallander, Green, Sugarman, \& Bavelier, 2011; Wu \& Spence, 2013). It also has been targeted as a method of cognitive behaviour training in healthy and patient populations (see Green \& Seitz, 2015). For instance, action-game training in healthy young adults, dyslexic children, and elderly populations led to improvements in attentional tasks such as visual search, focused attention, and divided attention (Belchior et al., 2013; Franceschini et al., 2013; Wu \& Spence, 2013). Previous studies in action gaming have typically measured visual attention by recording reaction time and accuracy of the manual responses in various attentional and perceptual tasks (e.g. Green \& Bavelier, 2006; HubertWallander et al., 2011; West, Stevens, Pun, \& Pratt, 2008). However, one of the core functions of visual attention is programming of saccadic eye movements to areas of the world that contain the information we need (e.g. Deubel \& Schneider, 1996; Hoffman \& Subramaniam, 1995; Kowler, Anderson, Dosher, \& Blaser, 1995). For example, facilitation of saccades (i.e. shorter saccade reaction time) has been observed when participants allocate attention to a target, participants show better perceptual identification at the saccade goal than elsewhere, and participants fail to dissociate the locus of attention and the saccadic endpoint (Hoffman \& Subramaniam, 1995; Kowler et al., 1995). The few studies 
that have investigated action-game-related changes in visual attention by recording saccadic eye movements have tended to use simple saccadic paradigms such as oculomotor capture task, saccadic trajectory paradigms, prosaccades, and antisaccades (see Chisholm \& Kingstone, 2012, 2015; Mack \& Ilg, 2014; West, Al-Aidroos, \& Pratt, 2013). Here, we look to extend this work to examine eye movements in more naturalistic tasks.

Comparisons of action gamers and nongamers in the aforementioned studies showed enhancements in action gamers, including fewer saccades towards distractors (Chisholm \& Kingstone, 2012, 2015), reduced saccadic deviation towards the distractors as saccade reaction time increased (West et al., 2013) and shorter latencies in double step prosaccade and antisaccade tasks (Mack \& Ilg, 2014; this article did not especifially recruited action gamers). However, it is still unclear how action game playing may affect managing the competition for attention across our visual world through eye movements (i.e. where people choose to look). If actionvideo-game playing enhances visual attention in a manner that affects selection of visual information, this has important implications for the utility of it in natural tasks performance. The existing game research literature can allow us to make a number of predictions about the ways in which eye movements might change.

\section{Fixation durations and saccade amplitudes}

Game-related improvements in information processing have been inferred from observing faster reaction times with equal accuracy in action video gamers than nongamers (Dye, Green, $\&$ Bavelier, 2009), showing enhanced contrast sensitivity (Li, Polat, Makous, \& Bavelier, 2009) and more efficient perceptual decision making (Green, Pouget, \& Bavelier, 2010). If this is evidence of a general ability to efficiently resolve more information in less time, we might expect there to be a decrease in fixation durations after action-game training as a consequence of enhanced visual processing during each fixation.

In contrast, given that action-game playing has also been associated with a larger useful field of view (Feng et al., 2007; Green \& Bavelier, 2003, 2006), suggesting that participants are able to process information from a wider area during each fixation (Ball, Beard, Roenker, Miller, \& Griggs, 1988), fixation duration might increase. If this occurs, participants may make fewer short-amplitude saccades, as they process information in the surrounding area during a fixation. Overall, this would lead to an average increase in saccade amplitude size. Another possibility is that increasing in information processing and useful field of view together might cause no change in the duration of fixations, as more information (from a wider area) is processed faster, so no change would be expected.

\section{Spatial distribution of fixations}

Generally, gaze tends to be directed to areas that will provide information for the task being completed (Buswell, 1935; Yarbus, 1967). Recently, emerging models of eye guidance have shown that understanding the way that targets tend to be arranged across a scene can guide visual search (Ehinger, Hidalgo-Sotelo, Torralba, \& Oliva, 2009; Kanan, Tong, Zhang, \& Cottrell, 2009; Torralba, Oliva, Castelhano, \& Henderson, 2006; Zelinsky \& Schmidt, 2009). For example, when wanting to know the time, people tend to search the walls for a clock, rather than the floor. Conversely, when searching for your shoes, the floor becomes a more likely place to locate them (see Torralba et al., 2006). Models attempting to understand fixation locations when searching for people have tended to be successful when incorporating some constraints on the likely locations of people (see Ehinger et al., 2009; Kanan et al., 2009), compared to models based on low-level visual features such as salience (e.g. Itti \& Koch, 2000). When people understand the underlying spatial distribution of targets, their search is directed towards these locations (Druker \& Anderson, 2010). Because part of the aim in action gaming is to find and eliminate targets, it is possible that gaming is simply accompanied by learning the spatial distribution of targets important to the game.

Clark, Fleck, and Mitroff (2011) compared action gamers and nongamers on a change-detection paradigm employing both change and no-change trials. They measured the distance between one click to the next in no-change trials when participants guessed the location of the change. Gamers showed larger movements from one point to the next. Moreover, gamers tended to cover all four quadrants of each image, whereas nongamers were more likely to click on the same quadrant several times, suggesting a tendency in gamers to search the entire area. They suggested that these findings were indicative of gamers employing a search strategy that was spread more widely across the stimuli. If a wider field of information is processed at each fixation point as a result of gaming, the eyes might move larger distances (i.e. making larger saccades); in this way, search would be similarly spread more widely, thereby monitoring the entire area. However, in the Clark et al. (2011) study, the target was not spatially predictable, and it is unclear whether or not prior knowledge about the possible distribution of the targets might override such wider distribution of search.

\section{Present study}

In this study we aimed to investigate the influence of action video gaming on eye movement behaviour while search in an abstract conjunctive search (relatively abstracted from natural behaviour), searching for a Gabor patch in game photographs 
and in real city-centre scenes (to investigate eye movement behaviour for searching for an arbitrary embedded object in a natural scene), a search requiring counting people within game photographs (a task with similar requirement to the game) and a more natural search requiring counting people in city street photographs (to investigate the influence of action game training on eye movement behaviour in natural scene). By approaching the question using tasks that are relatively abstracted from natural behaviour, to tasks more similar to those we have to conduct in our daily lives, we are able to start to examine whether findings might extend outside of the laboratory. We looked at fixation duration, saccade amplitude, and distribution of fixations before and after action videogame training. We also recruited 20 experienced action gamers and compared their performance in the same-search paradigms with nongamers' pretraining performance to investigate how substantially more experience with action games might influence eye movement behaviour. We therefore take the dual approaches of experimental training and observational study of the question.

While both are typically used in the video-game literature, each has advantages and disadvantages. With game training, effects are likely driven by the manipulation, with a control group determining whether effects are due to repeat testing, or the manipulation. However, it is difficult to determine whether null effects might be due to not enough training. Here, we used 10 hours as significant changes in perception have been found from this length of time (Feng et al., 2007; Green \& Bavelier, 2003, 2006; Wu \& Spence, 2013). Observational study allows us to examine much more gaming experience, but there are many factors that may differentiate gamers from nongamers, such as an existing propensity to playing games $d u e$ to better perceptual skills. By taking both approaches, we can examine which results are consistent across methodologies to try to gain a clearer picture of gaming effects.

\section{Experiment 1}

\section{Method \\ Participants}

Forty nongamers (20 males and 20 females) between 17 and 35 years old were recruited from the University of Melbourne. Nongamers had no experience of any video game and mobile game play in at least the last 2 years. All participants had normal or corrected-to-normal visual acuity as tested with a LogMAR chart at 3 meters and normal colour vision as tested by the Ishihara test. Nongamers were alternately assigned to the action-game training or card-game training groups as they were recruited. Each training group consisted of 20 participants (10 males and 10 females). Mean age was 25.75 years
$(S D=3.95)$ for the action-game training group and 26 years $(S D=5.32)$ for the card-game training group. Written consent was obtained from each participant prior to participation, and they were compensated AUD \$10 per session for their participation. The study was approved by the University of Melbourne Human Research Ethics Committee and conducted in accordance with the Declaration of Helsinki.

\section{Apparatus}

Eye movements were recorded using an Eyelink II head mounted eye tracker (SR Research). Viewing was binocular, but the left eye only was tracked as no disconjugacy was expected in normal subjects. Eye position was sampled at $500 \mathrm{~Hz}$ with spatial accuracy of $0.5^{\circ}$. The images were displayed using a short-throw projector (NEC, WT610) on a screen at a viewing distance of $130 \mathrm{~cm}$ and resolution of 1024 $\times 768$. Image presentation and eye tracking was controlled with SR Research Experiment Builder software.

Game training was performed using a 15.5-in. laptop (Dell, Latitude E6520) with $1920 \times 1080$ resolution for both training groups. The monitor subtended $35.3^{\circ} \times 19.6^{\circ}$ visual angle when the player sat $55 \mathrm{~cm}$ from the laptop- the approximate viewing distance for all participants.

\section{Stimuli}

Five different sets of images were prepared as stimuli for the search tasks. Images were of resolution $1024 \times 576$ pixels and subtended $62.8^{\circ} \times 38.2^{\circ}$ of visual angle on the screen. Examples of the stimuli are presented in Fig. 1. Forty conjunctive search task images were created by R statistical software (A language and environment for statistical computing, $\mathrm{R}$ Foundation for Statistical Computing, Vienna, Austria, http://www.R-project.org). Each image contained 59 red and green letter $d \mathrm{~s}$ and green $b \mathrm{~s}$ as distractors and one red letter $b$ as the target that were randomly positioned on a white background. Each letter subtended approximately $0.6^{\circ} \times 1^{\circ}$ degrees of visual angle. Then, we selected 80 photographs of city scenes and in-game photos. The city photographs were found using Google Image search, and the in-game images were screen-captured while one of the experimenters was playing through the game Call of Duty: Modern Warfare II (Infinity Ward). There were an average of $12.47(S D=5.56)$ people in the city images and $5.21(S D=2.8)$ in the game images. For the Gabor search tasks, a Gabor patch of $150 \times$ 150 pixels was inserted into each of the 80 city and game photographs using MATLAB (R2010b, The MathWorks, Inc., Natick, MA, U.S.). The location of the Gabor was randomly selected. Stimuli were piloted $(N=3)$ to ensure that all targets were detectable, if any of the targets could not be found by participants in the pilot study, the Gabor would be randomly positioned in another place in the photograph until all 

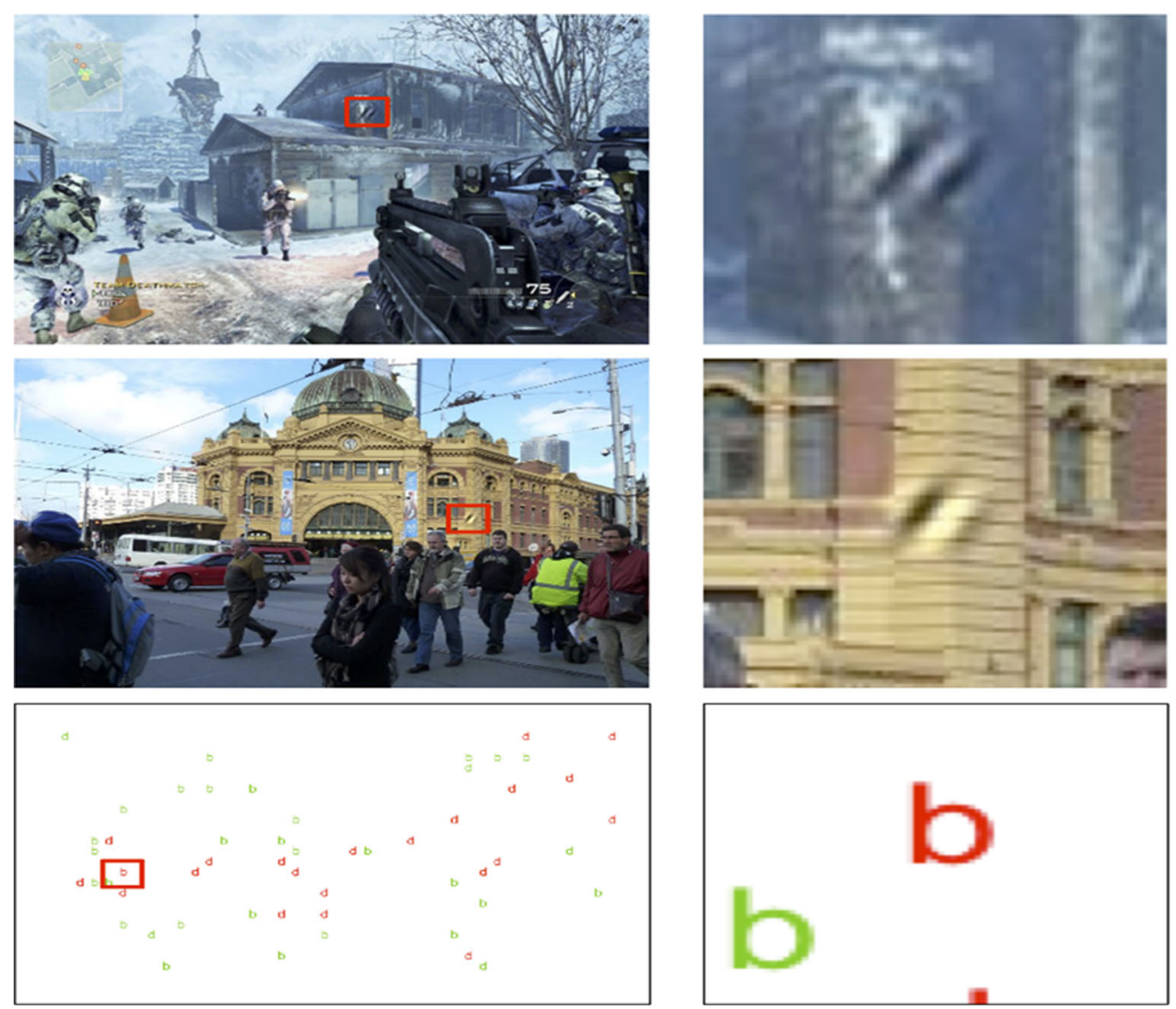

Fig. 1 Sample images from the three image types. Upper panelphotograph from Call of Duty: Modern Warfare II game with embedded Gabor target (magnified in the upper right panel). The Gabor was not present in the people-counting task. Middle panel-city

targets could be detected. Targets were uniformly distributed across all stimuli.

The 80 city and in-game photographs were randomly split into four sets (each contained 20 images) and two sets of abstract images. Each participant saw different set of images either in pre- or posttraining session (i.e. each participant saw each image only once), and the condition that the image was used in (people counting vs. Gabor search, pre- and posttraining) was counterbalanced across the experiments. The order that the images were presented in each testing session was randomised by the Experimental Builder software driving the Eye Link II eye tracker.

\section{Procedure}

Testing procedure Participants sat $130 \mathrm{~cm}$ from the screen while wearing the eye tracker (see Fig. 2). An automated 9point calibration and validation was performed before starting each task, with calibration being repeated if the spatial accuracy on validation was worse than $0.5^{\circ}$. Five different types of stimuli were shown to the participants while recording their eye movements. To familiarise participants with each task, they completed five practice trials before starting that task. street photograph with embedded Gabor. Bottom panel - abstract search image with singleton red $b$ target magnified on the right (Colour figure online)

In each task, 20 images on the screen, one at a time. Each image stayed on the screen until the subject's reached their decisions (indicated by pressing a button on a gamepad to end the trial) or until an automatic time-out of 20 seconds. In the abstract search task, the participants' task was to find a single red $b$ and then press the button on the gamepad as fast and accurately as possible. In the people-counting task, participants were asked to count the number of people that were in the picture and then push the button on the gamepad to end the trial and report their answer to the experimenter. In the Gabor search task, participants were asked to respond as soon as they found the single Gabor patch embedded in the photograph.

Training procedure Participants were randomly assigned to either the action-game training group, who were asked to play a first-person shooter game (FPS) Call of Duty (COD): Modern Warfare II or to the card-game training group, who were asked to play the card game Free Cell (Microsoft Windows). They played the game assigned for a total of 10 hours over two weeks for 1 hour a day. We chose to have an active control group to equalise the level of motivation and engagement between groups to remove any Hawthorne effect (wherein individuals who have been under scrutiny 
Fig. 2 A participant while doing the city Gabor task

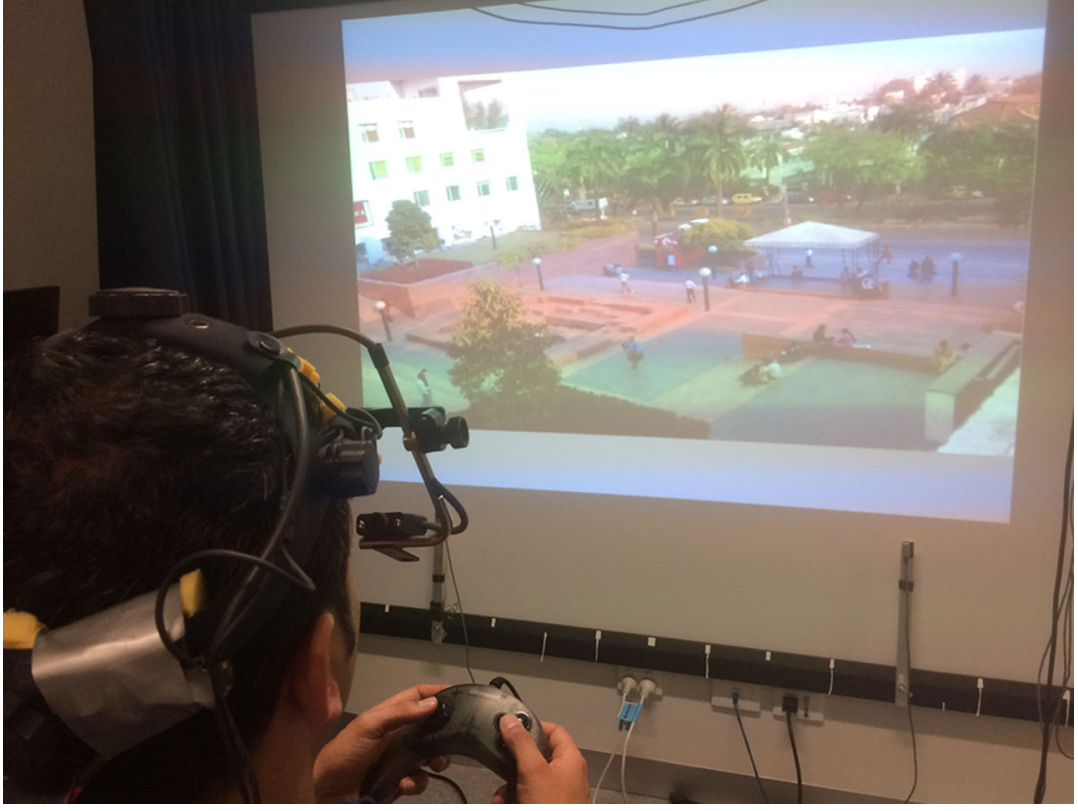

outperform others in whom no such interest is taken) and to monitor for any test-retest effect (Boot, Blakely, \& Simons, 2011). A card game was selected as the control game as, despite it involves some level of search to find the target and sort the cards, it still makes low attentional demands in that it does not require divided attention, constant alertness, tracking of multiple objects, and fast responses to sudden events, the characteristics that a FPS game usually contains (Cohen, Green, \& Bavelier, 2007).

In the first session of the action-game training the content and aim of the game, different movements and weapons were explained to the trainee. They started playing the game by completing the first mission, which serves as a tutorial for the player. After finishing their daily training, their achieved level was recorded such that if they could not finish a mission completely, they started from that uncompleted mission the next day. The action training group played the single-player mode of the game and each mission constituted a level. Because some of the trainees finished the single-player mode before the end of 10 training hours, they started to play the advanced Special Ops section in the game which was distributed over five categories. In total, there were 17 missions in the singleplayer mode and five from Special Ops.

The aim of Free Cell is to sort the cards from Ace to King in order for the same colour and the same shape, liberating 'trapped' cards that lie under nonconsecutive cards. Participants were encouraged to complete as many games as they could during the training session. The number of games played, games won, wins percentage, longest winning streak, longest losing streak, and current streak were recorded.

\section{Analysis}

Saccades and fixations were defined using the saccade detection algorithm supplied by SR Research. All data were analysed in R. Median fixation duration and saccade amplitude were used in view of the skewed distributions of the variables. All dependent variables were analysed with $2 \times 2$ mixed ANOVA, with group (action game training group or card game training group) as between and session (pre or post) as within-subjects factors. Further $t$-test analysis with Bonferroni correction was also performed when appropriate.

\section{Results}

\section{Game performance}

To ensure that participants learned and were involved in their games, we performed a linear regression on the performance of each trainee from Day 1 to Day 10. The level achieved in the game (which can indicate the progress in the game) improved significantly in the action game training $\left(R^{2}=0.9, p<\right.$ $.001)$ and the game win percentage similarly improved $\left(R^{2}=\right.$ $0.5499, p=.01)$ in the control group. While these results are not directly comparable, they serve to demonstrate that participants' gaming ability increased across the training days.

\section{Accuracy of responses}

To assess how accurate performance was in the personcounting tasks, we calculated the number of people in each image that was over- or undercounted. We made these all positive as to examine absolute error (as a participant overcounting 
Table 1 Absolute error size of responses in counting tasks in each training group before and after the training

\begin{tabular}{llllll}
\hline & \multicolumn{2}{l}{ Action training group } & & \multicolumn{2}{l}{ Card training group } \\
\cline { 2 - 3 } \cline { 6 - 7 } \cline { 5 - 6 } & Pre & Post & & Pre & Post \\
\hline City count & 1.39 & 1.46 & & 1.38 & 1.56 \\
Game count & 0.94 & 1.14 & & 0.9 & 2.23 \\
\hline
\end{tabular}

by 1 half of the time, and undercounting by 1 on the other half would give the average score of perfect performance). We therefore subtracted the number of people reported in each image from the actual number of people in each image, and took absolute error from this (see Table 1). Statistical analysis on the error size in city people-counting task did not show main effects of the group ( $p=.6$ ), session $(p=.2)$, and no interaction was observed $(p=.6)$. However, in the game people-counting task, significant main effects of the group and session and a significant interaction was observed ( $p$ s $<$ .001 ), showing an increase in the error size in both groups.

For the Gabor search tasks, eye movements were manually checked to ensure that when participants' reported finding the target, the last fixation before ending the trial was on the location of the Gabor.

\section{Fixation duration}

Fixation duration for each task was calculated and compared between training groups and across testing sessions (see Fig. 3). There was no significant main effect of the group or session and also no interaction was observed for any of the tasks $(p>.1)$, except a significant interaction between group and session in the game Gabor search task, $F(1,38)=6.38, p$ $=.015$. Further $t$-test comparisons showed that card gamers' fixation durations became shorter, $t(38)=-1.9, p=.049$, from before to after the training. But no significant changes were observed in the action game training group, $t(38)=0.48, p=$ .17 , and no significant difference between groups was observed in Session 1, $t(34.88)=0.28, p=.7$, or in Session 2, $t(29.46)=-1.67, p=.105$.

\section{Saccade amplitude}

Saccade amplitude in each task was compared between training groups and across testing sessions (see Fig. 4). There were no main effects of the session or group and no significant interaction between factors in abstract, city people-counting task, city, and game Gabor task $(p>.1)$. However, in the game people-counting task, there was a significant main effect of the

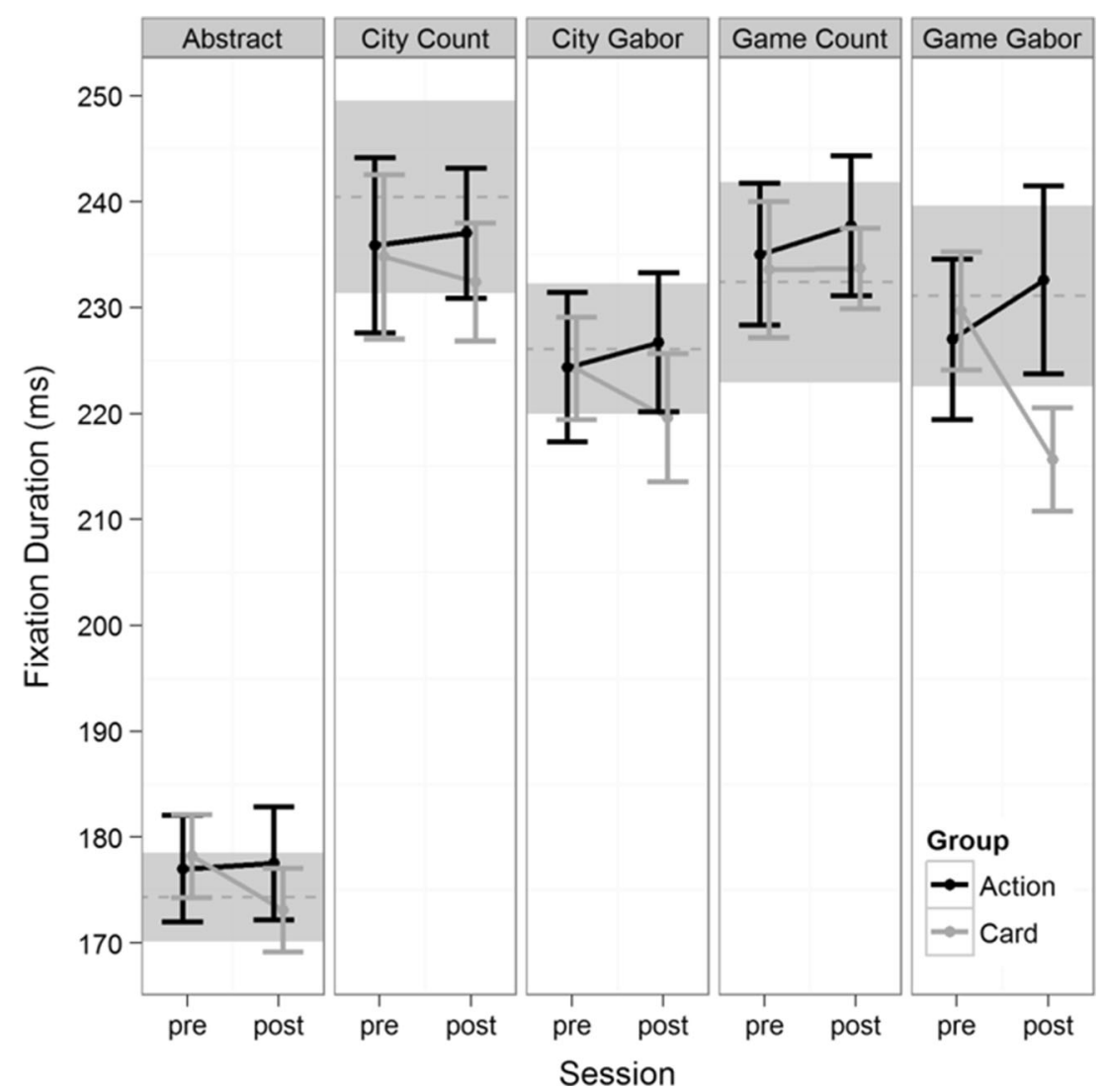

Fig. 3 Fixation duration (ms) across tasks, sessions, and in two training groups. Error bars show the standard error of the mean. The grey dashed line and grey background in each task show the mean and standard error of mean for the experienced action gamers (from Experiment 2) 


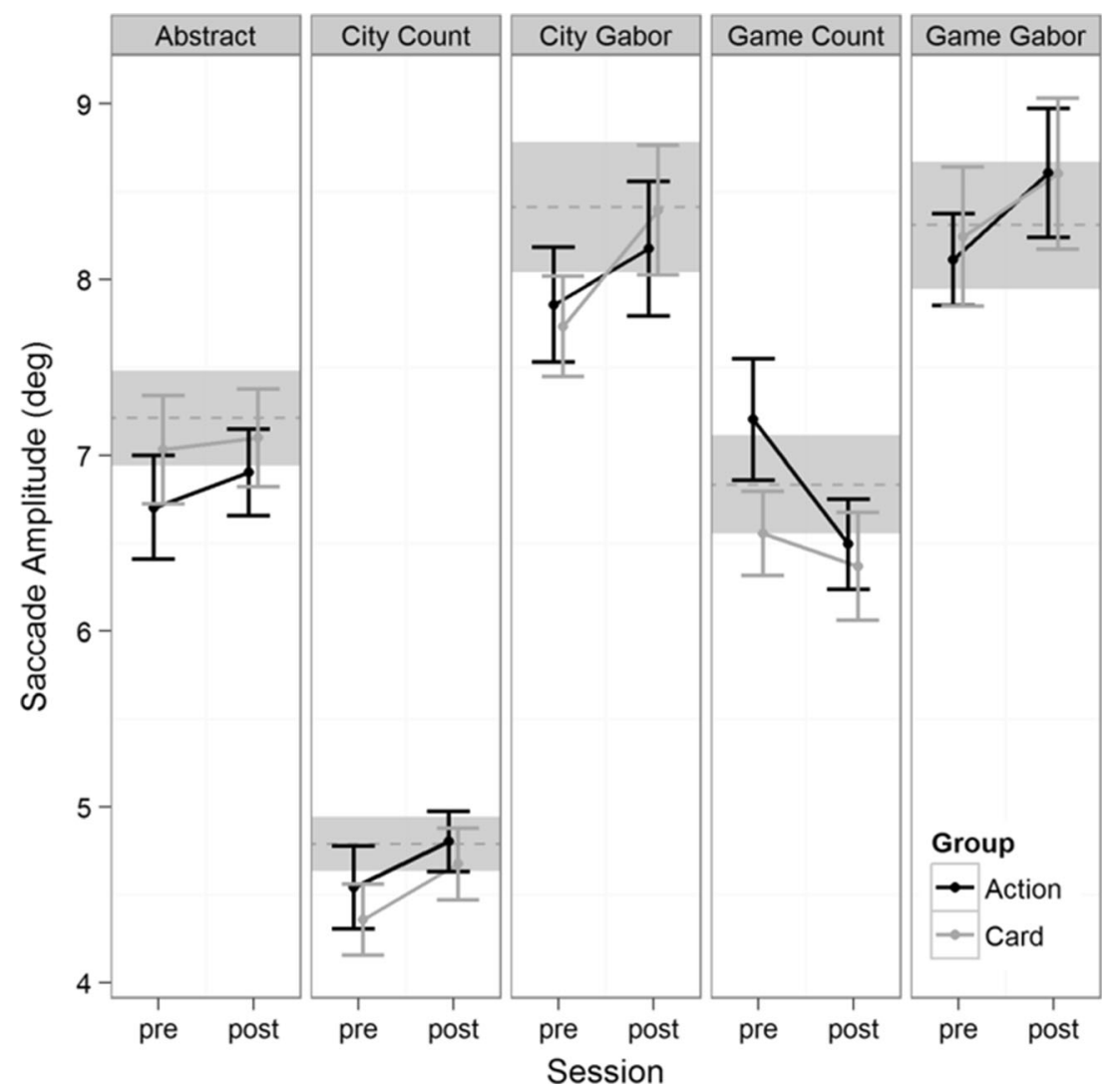

Fig. 4 Saccade amplitude (degrees of visual angle) across tasks, sessions, and in two training groups. Error bars show the standard error of the mean. The grey dashed line and grey background in each task show mean and standard error of the mean in experienced action gamers (from Experiment 2)

session, $F(1,38)=4.19, p=.047$, but no main effect of the group $(p=.27)$ and no interaction was observed $(p=.23)$. Further $t$-test analysis showed a trend toward a decline in saccade amplitude in the action game training group, $t(38)=$ $-1.65, p=.06$, but no significant change in the control group $(p$ $=.47$ ). Moreover, groups were not significantly different before, $t(33.94)=-1.54, p=.13$, or after the training, $t(36.84)=-$ $0.3, p=.75$.

\section{Correlational analysis for changes in fixation duration and saccade amplitude}

To investigate whether or not there is a trade-off or any relationship between changes in fixation duration and saccade amplitude (from pre- to posttraining), we correlated changes in the two variables in all five search tasks in both groups. There was no significant relationship between these two variables in any of the groups $(p>.1)$.

\section{Spatial distribution of fixations}

To examine whether the spatial allocation of foveal gaze changed for the training groups across testing sessions, we examined the spread of horizontal and vertical distributions of fixations by analysing the standard deviation of fixated locations along both axes.

The horizontal distribution of fixations did not show any changes across sessions and between groups in any of the tasks $(p>.05)$. Analysing vertical distribution of fixations showed no main effect of the group, session, and no interaction in the abstract and city people-counting tasks $(p>.1)$. However, in the game people-counting task, there was a trend towards significant main effect of the group, $F(1,38)=2.87$, $p=.09$, and a significant interaction between session and group, $F(1,38)=4.87, p=.03$, although no main effect of the session $(p=.1)$ was observed. When examined further, there was a significant reduction in the vertical distribution after the training in the action game group, $t(38)=-1.81$, $p=.02$, from pre- to posttraining, but not in the card group $(p=.4)$. Moreover, groups became significantly different in the posttraining session, $t(37.38)=2.72, p=.009$. In the game Gabor search task, there was also a main effect of the group, $F(1,37)=5.059, p=.03$, and session, $F(1,37)=5.35$, $p=.026$, with the action game training group showing a small, but significant reduction in distribution of fixations, $t(38)=-2.22, p=.047$; however, card gamers did not show a significant change, $t(38)=-0.925, p=.24$. No significant interaction between session and group was observed. In the 


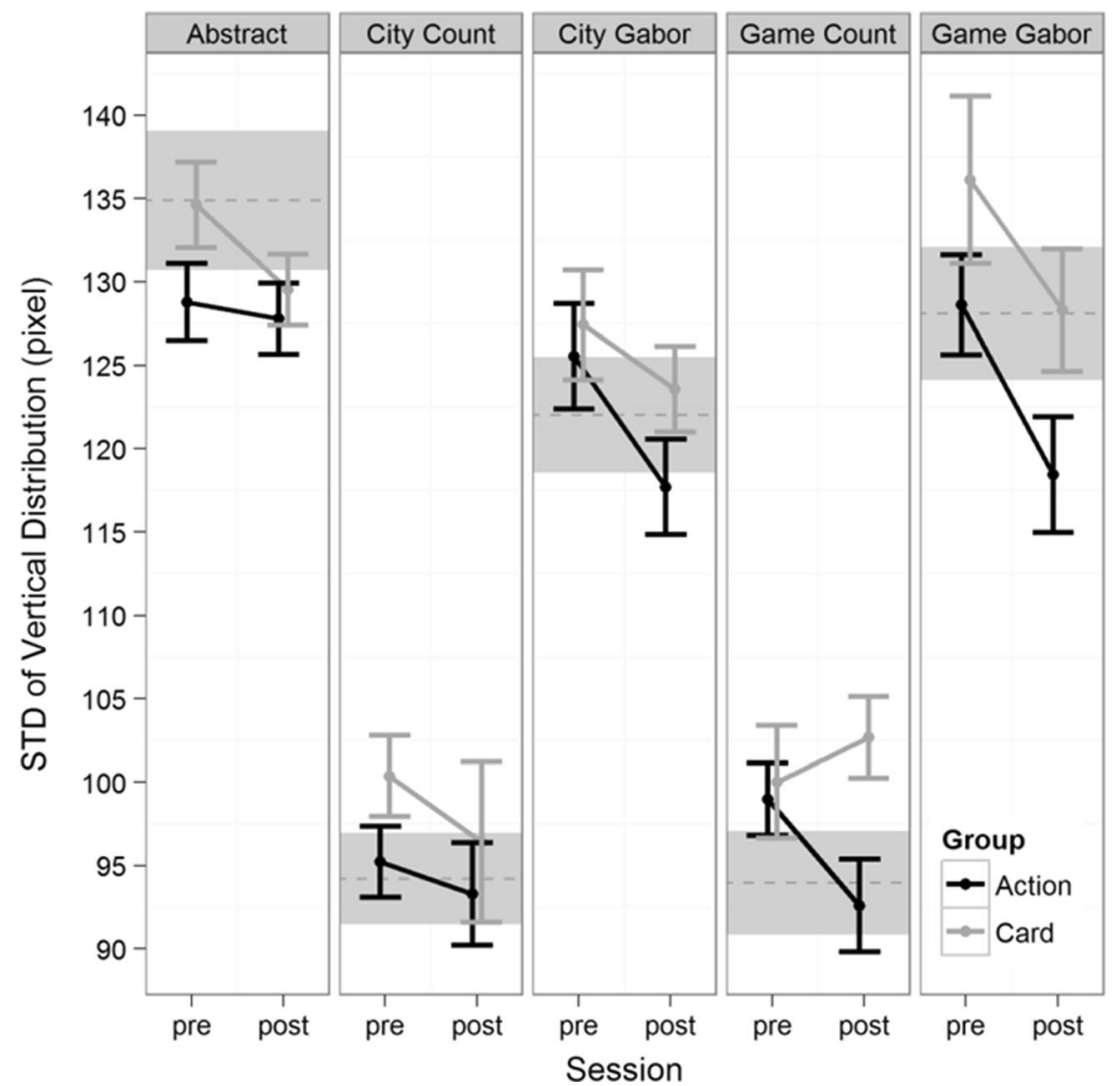

Fig. 5 Vertical distribution of fixations (in pixels) across tasks, sessions, and in two training groups. Error bars show the standard error of the mean. The grey dashed line and grey background in each task show the

mean and standard error of the mean of vertical distribution of fixations in experienced action gamers (from Experiment 2)

city Gabor task also there was a significant main effect of the session, $F(1,38)=7.82, p=.008$, with action game training group showing a significant reduction in the distribution of fixations from before to after the training, $t(38)=-1.84$, $p=.009$ (see Fig. 5).

One potential explanation for reduction in the vertical spread of fixations in the game-counting task is that it might have been confounded by the target locations. Does this

change in distribution reflect a change in general search behaviour, or could it be that the action training group learned the distribution of targets? Figure 6 shows that when we plotted the distribution of people in the counting images, targets tended to be in a horizontal band that is similar in both the city and game photographs. This band likely represents the ground or horizon, where targets are most likely to be (see Ehinger et al., 2009, for example).
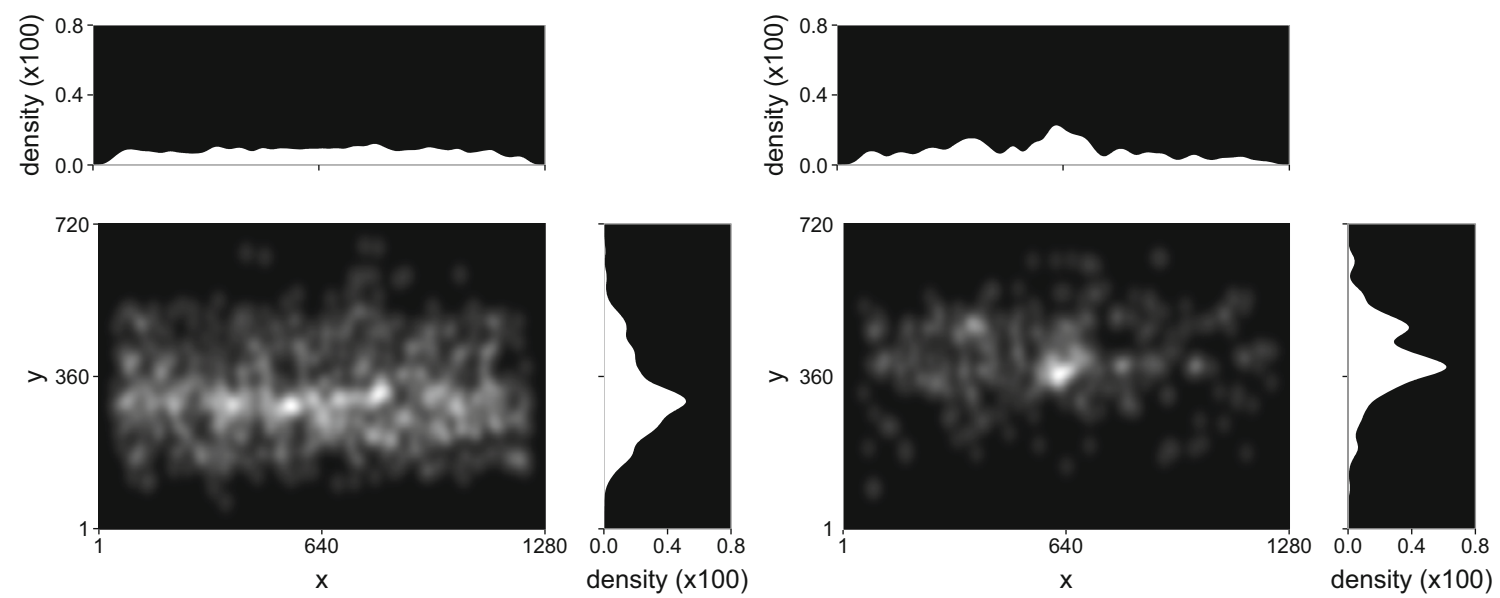

Fig. 6 Target distribution in both city (left panel) and game (right panel) photographs 


\section{Interim discussion}

It has been suggested that gaming can modify several aspects of visual attention. Here, we investigated how action game training affects eye movement behaviour (i.e. overt attention allocation) during visual search of an abstract conjunctive search task of computer game scenes and of natural scenes. Our findings suggested that the action-game training group developed a narrower search pattern when counting the number of soldiers in the game photographs, with a reduced vertical distribution of fixations when looking for soldiers. The reduced vertical distribution of gaze in the game peoplecounting task alone suggests that the action-gaming group was learning where to look for the targets in the game (i.e. enemies), developing a 'contextual guidance' for game images (Ehinger et al., 2009; Kanan et al., 2009). However, they did not search natural images differently. There were not any other modifications in duration of fixations or saccade amplitudes.

Previous studies have used 10 hours of action video-game playing and revealed changes in visual attention (Belchior et al., 2013; Feng et al., 2007; Green \& Bavelier, 2003, 2006; Wu \& Spence, 2013). However, to ensure that our results are not related to the amount of training that we gave to the participants, we ran a control experiment, where we compared a group of experienced action gamers to our nongamers before the latter group completed the training. Although it is not possible to demonstrate causation of video-game playing in such an observational comparison (i.e. are changes in performance due to extensive video-game playing, or are those who choose to play, and continue to play games are individuals with preexisting higher perceptual or motor skills?), we can at least examine whether those who have a lot of experience playing action video games show different saccadic eye movement characteristics than those who do not.

\section{Experiment 2}

\section{Method}

\section{Participants}

Along with the pretraining data from the 40 nongamers from Experiment 1 , aged 25.87 years $(S D=4.63)$, we collected data from 20 experienced action gamers (16 males and 4 females) between 17 and 28 years old, with a mean age of 23.2 years $(S D=4.09)$. Our experienced gamers reported $10.05(S D=$ 4.02) hours per week of playing shooting, multiplayer online battle arena, action role playing, or action adventure games in the last 2 years. They also had the experience of playing Call of Duty, Modern Warfare II (our training game). All of the experienced gamer participants showed normal or corrected- to-normal visual acuity and normal colour vision tested in the same manner as Experiment 1, and provided informed consent before taking part. They were compensated AUD \$10 for their time.

\section{Apparatus, stimuli, and procedure}

Experienced action gamers carried out the same five tasks as described in Experiment 1-conjunctive abstract search, Gabor search in game and city photographs, and people search in game and city photographs. The protocol for testing was identical to Experiment 1, except that the experienced gamers did not undergo the training and were only tested once. Stimulus presentation was counterbalanced such that all stimuli were presented equally across the experienced gamer group, with city and game photographs being used for the Gabor and people search an equal number of times across the experiment.

\section{Analysis}

Experienced action gamers and nongamers were compared using independent-samples $t$ tests.

\section{Results}

Accuracy of responses

Similar to Experiment 1, the absolute error size for each trial was calculated and then averaged across trials in each group (see Table 2). Independent-samples $t$ tests did not show any significant differences between groups in any of the tasks $(p>.1)$.

Similar to experiment 1 in Gabor search tasks, eye movements were manually checked to ensure that when participants' reported finding the target, the last fixation before ending the trial was on the location of the Gabor.

\section{Fixation duration}

Fixation duration for the experienced action gamers was not significantly different from trainees either before (see Table 3) or after the training (see Fig. 2), suggesting that neither game training nor longer and more extensive prior gaming experience appeared to affect duration of fixations.

Table 2 Absolute error size of responses in counting tasks in experienced action gamers and nongamers (pretraining)

\begin{tabular}{lll}
\hline & Nongamers & Experienced action gamers \\
\hline City count & 1.39 & 1.48 \\
Game count & 0.92 & 0.96 \\
\hline
\end{tabular}


Table 3 Comparison of fixation duration ( $\mathrm{ms}$ ) between nongamers and experienced action gamers in five search tasks

\begin{tabular}{lrlll}
\hline & $\begin{array}{l}\text { Nongamers } \\
(\text { mean } \pm S D)\end{array}$ & $\begin{array}{l}\text { Experienced action gamers } \\
(\text { mean } \pm S D)\end{array}$ & $t$ & $p$ \\
\hline Abstract & $177.6 \pm 19.89$ & $174.33 \pm 18.87$ & 0.18 & 0.85 \\
CP & $235.33 \pm 35.53$ & $240.45 \pm 40.73$ & 0.479 & 0.63 \\
GP & $234.3 \pm 29.02$ & $232.4 \pm 42.3$ & -0.17 & 0.85 \\
CG & $224.32 \pm 26.66$ & $226.12 \pm 27.61$ & 0.24 & 0.81 \\
GG & $228.33 \pm 29.29$ & $231.11 \pm 38.05$ & 0.28 & 0.71 \\
\hline
\end{tabular}

Note. $\mathrm{CP}=$ city people-counting task; GP = game people-counting task; $\mathrm{CG}=$ city Gabor search task; $\mathrm{GG}=$ game Gabor search task

\section{Saccade amplitude}

There was no significant difference in saccade amplitude between experienced action gamers and nongamers either before (see Table 4) or after the training (see Fig. 3) in any of the tasks.

\section{Spatial distribution of fixations}

Changes in the vertical distribution of fixations between nongamers pretraining and experienced action gamers were compared. As Table 5 shows, there is no significant difference between groups in any of the tasks.

\section{General discussion}

We found no changes in eye movement behaviour after 10 hours of video-game training, except that participants who played action games seemed to have a different strategy for searching action-game images for game-relevant targets. We conducted an observational control experiment to examine whether those with substantially more experience of action games than our 10 hours of training showed any differences in eye-movement behaviour than our nongamers before they underwent training. One argument that could be levelled

Table 4 Comparison of saccade amplitude (deg) between nongamers and experienced action gamers in five search tasks

\begin{tabular}{lllll}
\hline & $\begin{array}{l}\text { Nongamers } \\
(\text { mean } \pm S D)\end{array}$ & $\begin{array}{l}\text { Experienced action gamers } \\
(\text { mean } \pm S D)\end{array}$ & $t$ & $p$ \\
\hline Abstract & $6.86 \pm 1.33$ & $7.21 \pm 1.7$ & 1.007 & 0.31 \\
CP & $4.45 \pm 0.97$ & $4.78 \pm 0.68$ & 1.54 & 0.12 \\
GP & $6.87 \pm 1.35$ & $6.83 \pm 1.25$ & -0.12 & 0.89 \\
CG & $7.79 \pm 1.355$ & $8.41 \pm 1.66$ & 1.44 & 0.15 \\
GG & $8.17 \pm 1.47$ & $8.3 \pm 1.61$ & 0.30 & 0.76 \\
\hline
\end{tabular}

Note $. \mathrm{CP}=$ city people-counting task; $\mathrm{GP}=$ game people-counting task; $\mathrm{CG}=$ city Gabor search task; $\mathrm{GG}=$ game Gabor search task
Table 5 Comparison of standard deviation of vertical distribution of fixation (in pixels) between nongamers and experienced action gamers in five search tasks

\begin{tabular}{lrrrr}
\hline & $\begin{array}{l}\text { Nongamers } \\
(\text { mean } \pm S D)\end{array}$ & $\begin{array}{l}\text { Experienced action gamers } \\
(\text { mean } \pm S D)\end{array}$ & $t$ & $p$ \\
\hline Abstract & $131.69 \pm 11.17$ & $134.88 \pm 18.73$ & 0.699 & 0.49 \\
CP & $97.8 \pm 10.39$ & $94.2 \pm 12.26$ & -1.12 & 0.26 \\
GP & $99.49 \pm 12.55$ & $93.96 \pm 13.98$ & -1.49 & 0.14 \\
CG & $126.47 \pm 14.27$ & $122.03 \pm 15.54$ & -1.07 & 0.29 \\
GG & $132.27 \pm 18.21$ & $128.09 \pm 17.77$ & -0.84 & 0.4 \\
\hline
\end{tabular}

Note. $\mathrm{CP}=$ city people-counting task; $\mathrm{GP}=$ game people-counting task; $\mathrm{CG}=$ city Gabor search task; GG = game Gabor search task

against us from Experiment 1 alone is that the amount of training was not sufficient. Experiment 2 would suggest that not to be the case, as we find the same results. One argument that could be levelled against us from Experiment 2 alone is that gamers may differ on more than just the fact that they play games (perhaps they have inherently better attentional skills). Experiment 1 provides the necessary control to rule out that possibility. The only difference we found in Experiment 1 that we did not find in Experiment 2 was the reduction in vertical distribution of gaze. This may be due to the specificity of training that we gave our action gamers. Our experienced gamer group had played many different action games, whereas our training group had only played Call of Duty, and played it for an hour, every day, for 10 days. Action gaming may have taught our training participants a very specific contextual cue to search for people, one that is relevant only for this game. For example, if an entirely naive person learned to search for a cup in a kitchen, much of that knowledge would not help them find a cup in a lounge (other than having the same physical rules such as gravity). Therefore, our effect may be more finegrained in our training group, where experienced gamers can use their knowledge of all games to cue search performance.

Fixation durations, saccade amplitudes and spread of fixations were indistinguishable between action gamers and nongamers. While increased processing speed and larger useful field of view have been shown in action gamers (e.g. Feng et al., 2007; Green et al., 2010), extensive prior action-gaming experience failed to lead to any changes in the duration of fixations or saccade amplitude in our paradigms. This would suggest that the covert selection processes that have been shown to change with action gaming do not influence overt selection. Covert selection has an important role in overt selection - the decision of where to move the eyes is a competition between all locations in the visual periphery, with covert attentional allocation playing some role in guiding the eye to sensible locations. Either the skills that can be trained with action gaming are not powerful enough to influence this decision, or these skills are not used when deciding where to move the eyes. However we did find a narrower distribution of 
fixations after action game training in the most similar task to the game which has been discussed below.

\section{Developing a narrower search strategy?}

The decrease in the vertical distribution of fixations after training in the game people-counting task and when searching for Gabor patches in both the city and game images in the action training group suggests development of a generally narrower spatial allocation of gaze during search, which is inconsistent with our wider search hypothesis based on developing wider useful field of view after action-game training. However, there are many other contributing factors that need to be taken into account. First of all is the difference in the search requirements between the game people-counting task and finding a randomly embedded Gabor patch in the city and game images. In searching for people in the game photographs for instance, what drives the gaze is the generic knowledge about the scene and understanding the possible arrangement of the targets (Ehinger et al., 2009; Kanan et al., 2009; Torralba et al., 2006; Zelinsky $\&$ Schmidt, 2009); however, in searching for a Gabor patch in the city and game images, such a priori knowledge cannot help. Therefore, the narrower searches during the posttraining session in the action-game training group in game people-counting task and in the search for Gabor should be investigated separately.

In explaining the narrower search in the game peoplecounting task, learning about the spatial location of targets seems likely, as it could be attributed to the repeated exposure to scenes of this type as a result of playing the game (Brockmole \& Henderson, 2006; Eckstein, Drescher, \& Shimozaki, 2006) and to the influence of objects appearing at consistent locations (Oliva, Torralba, Castelhano, \& Henderson, 2003; Torralba et al., 2006) rather than game training per se. Thus, it would appear that the skills required for learning to play a FPS action game (i.e. fixate and engage enemies) simply taught people how to look for people in those scenes.

Similar learning of spatial biases in target location has been shown by Druker and Anderson (2010), where participants responded faster to targets that appeared in an uncued 2-D distribution of targets that was biased towards an off-centre position. Similarly, Jones and Kaschak (2012) revealed that more first saccades were made to high probability locations during the search for a target. In this context it would be interesting to know if playing a game where targets were most likely to be present elsewhere (e.g. in a simulation of skeet shooting) the fixation distribution shifted to the relevant screen region. These factors may be underlain by a fixation decision mechanism whereby areas of high target probability have increased sensitivity, as demonstrated by Carpenter and Williams (1995). They reported that saccade latency was related to the probability of appearance of a target in a simple step task. Anderson and Carpenter (2006) also showed that this mechanism can explain changes in saccadic latencies when spatial biases are changed during a trial, with people gradually using new information about target locations to update their spatial biases (consistent with changes in Carpenter and Williams's, 1995, LATER decision-making model). These experiments would suggest that across time, we are able to learn where we are likely to find targets and adjust our search behaviour accordingly, either by executing more saccades to these locations or becoming more sensitive to targets presented there. Such a mechanism would certainly account for why we found changes in search behaviour in game images contingent on experience with the game. This has also been supported by the differences in fixation strategies of experts compared to novices in activities such as sport (Land \& McLeod, 2000), driving (Land \& Lee, 1994), and when searching medical images for signs of disease (Donovan \& Litchfield, 2013; Stainer, Anderson, \& Denniss, 2015). In further support of this, our nongamer group, after training on the action game, became more similar to our experienced action gamers in terms of the vertical distribution of gaze from exposure to the game, with no such change being seen in the card game-training group, who had no exposure to the game other than that of the experimental trials. Moreover, the action game training group showed a trend towards making smaller saccades after training on the same task. This might have occurred due to searching in a horizontal band of each photograph, where targets were mostly distributed.

But what caused a narrower search in the action game training group in the Gabor tasks? Figure 4 shows a reduction (although not statistically significant) in the distribution of fixations in the control group as well. This reduction in the control group might indicate a practice effect or test-retest effect as a result of repeated exposure to the task (Boot et al., 2011; Kristjánsson, 2013).

Our experienced action gamers in Experiment 2 also did not show a wider search strategy in comparison to the nongamers before the training in any of the tasks, even those with no contextual guidance (e.g. Gabor and abstract search). This somewhat contrasts with Clark et al. (2011), who found a wider search strategy in action gamers in a change detection task. However, they discussed their results based on data collected from mouse clicks as opposed to eye movements. Mouse clicking shows the final selected position, which might not take into account all of the intermediate locations that were examined, but which participants did not choose to select with a click. Our fixation analysis presented a more finely grained measure of attention allocation across a whole trial, and we found no difference in the spread of search compared to nongamers.

\section{No change in inspection of natural scenes}

In spite of the similar distribution of people in city and game photographs (in a middle horizontal band in the pictures), the 
decreased spatial distribution of fixations observed for game pictures was not replicated for city photographs (natural scenes). Eye-movement patterns are learned in natural tasks such that almost all the fixations are on the relevant objects (Hayhoe \& Ballard, 2005; Land, 2004). In other words, realworld tasks are accompanied by already learnt high-level contextual cues such as scene context (Mackworth \& Morandi, 1967) and task requirements (Rothkopf, Ballard, \& Hayhoe, 2007) which guide eye movements to relevant locations - for example, to the streets, sidewalks, and cars relevant to our task. Therefore, the absence of a change in scanning after game training may be due to there being nothing more to be learnt from the game that could facilitate natural scene viewing above the high-level ability we have already gained from day-to-day experience. Similarly, Gaspar et al. (2014) did not find any advantage of gaming on a real-world divided attention task such as street-crossing accompanied by performance of a working-memory task. Action gamers and nongamers experienced similar costs when crossing the street in a dual task condition. However, Chisholm and Kingstone (2015) showed less oculomotor capture (i.e. more accuracy at looking at the target rather than a distractor) in action gamers when looking at schematic faces (as socially meaningful stimuli) instead of abstract, socially neutral stimuli such as circles or dots. However, this might still be the result of enhanced top-down allocation of attention (see Chisholm \& Kingstone, 2012) rather than a transfer from the action game experience to performance in an oculomotor capture task in the context of face stimuli.

The abstract search paradigms that have typically been employed in the gaming literature so far (e.g. Castel, Pratt, \& Drummond, 2005; Hubert-Wallander et al., 2011) prohibit searchers from having a priori knowledge of where a target might appear. While this abstract approach is beneficial to control for such contextual guidance, the use of learntcontextual relationships is inescapable in most aspects of natural behaviour, with the exception perhaps of those individuals with memory deficits (e.g. Chun \& Phelps, 1999). It thus becomes more difficult to extrapolate from gaming-related effects on these more abstract tasks to how real-world behaviour might be affected.

Future studies may concern this issue by investigating any video-game benefits in more natural settings as this is an area of research that is relatively lacking in the current literature. The factor that is often neglected is that almost all of the studies have investigated the influences of game playing on measures that are relatively abstracted from naturally occurring tasks, which might not necessarily capture the experience of vision outside of the laboratory settings (see Kingstone, Smilek, \& Eastwood, 2008). For example, while findings indicate gaming is associated with a faster search time for finding a letter among distractors (Castel et al., 2005; HubertWallander et al., 2011), it is unclear from this whether we should expect participants to similarly show differences in the manner in which they search for a friend in a crowd of people.

One potential limitation of our study is that we reused data from Experiment 1 in Experiment 2. It is possible that if this data is in itself not representative of the true underlying eyemovement behaviour of nongamers, that conclusions from both experiments are drawn from flawed data. We would argue that while we cannot entirely rule this out, that the similarity between measures in the pre- and posttesting phases in Experiment 1 (except for the result that we have discussed here regarding distribution of gaze), and the similarity between the groups would make this unlikely.

\section{Conclusion}

This study examined whether there are changes in overt attention allocation associated with action-game training, and found no action-game related modifications to eyemovement characteristics except for a reduced spatial distribution of fixations while searching in game images. This may have represented learning the distribution of the targets (i.e. enemy soldiers) in game photographs, as there was no extension to a similar search for people in more natural, city centre scenes, a type of scene many of us likely encounter on an almost daily basis. While game training has been suggested as an enjoyable means for improving many facets of visual cognition, it is crucial to consider whether these changes might still affect behaviour once participants leave the laboratory and use vision in its natural context; the real world. Given the crucial role of eye movement in exploring our surroundings, and the lack of differences between action gamers and nongamers, further work should seek to align overt and covert selection in more natural paradigms.

Acknowledgements This work was supported by Albert Shimmins Postgraduate Write-up Award granted to E. Azizi.

\section{References}

Anderson, A. J., \& Carpenter, R. H. S. (2006). Changes in expectation consequent on experience, modeled by a simple, forgetful neural circuit. Journal of Vision, 6(8), 5.

Ball, K. K., Beard, B. L., Roenker, D. L., Miller, R. L., \& Griggs, D. S. (1988). Age and visual search: Expanding the useful field of view. Journal of the Optical Society of America A, 5(12), 2210-2219.

Bejjanki, V. R., Zhang, R., Li, R., Pouget, A., Green, C. S., Lu, Z., \& Bavelier, D. (2014). Action video game play facilitates the development of better perceptual templates. Proceedings of the National Academy of Sciences, 111(47), 16961-16966.

Belchior, P., Marsiske, M., Sisco, S. M., Yam, A., Bavelier, D., Ball, K., \& Mann, W. C. (2013). Video game training to improve selective 
visual attention in older adults. Computers in Human Behavior, 29(4), 1318-1324.

Boot, W. R., Blakely, D. P., \& Simons, D. J. (2011). Do action video games improve perception and cognition? Frontiers in Psychology, 2, 226.

Brockmole, J. R., \& Henderson, J. M. (2006). Using real-world scenes as contextual cues for search. Visual Cognition, 13(1), 99-108.

Buswell, G. T. (1935). How people look at pictures. Chicago, IL: University of Chicago Press.

Cain, M. S., Landau, A. N., \& Shimamura, A. P. (2012). Action video game experience reduces the cost of switching tasks. Attention, Perception, \& Psychophysics, 74(4), 641-647.

Carpenter, R. H. S., \& Williams, M. L. L. (1995). Neural computation of $\log$ likelihood in control of saccadic eye movements. Nature, 377(6544), 59-62.

Castel, A. D., Pratt, J., \& Drummond, E. (2005). The effects of action video game experience on the time course of inhibition of return and the efficiency of visual search. Acta Psychologica, 119(2), 217-230.

Chisholm, J. D., \& Kingstone, A. (2012). Improved top-down control reduces oculomotor capture: The case of action video game players. Attention, Perception \& Psychophysics, 74(2), 257-262.

Chisholm, J. D., \& Kingstone, A. (2015). Action video game players' visual search advantage extends to biologically relevant stimuli. Acta Psychologica, 159, 93-99.

Chun, M. M., \& Phelps, E. A. (1999). Memory deficits for implicit contextual information in amnesic subjects with hippocampal damage. Nature Neuroscience, 2(9), 844-847.

Clark, K., Fleck, M. S., \& Mitroff, S. R. (2011). Enhanced change detection performance reveals improved strategy use in avid action video game players. Acta Psychologica, 136(1), 67-72.

Cohen, J. E., Green, C. S., \& Bavelier, D. (2007). Training visual attention with video games: Not all games are created equal. In H. F. O'Neil \& R. S. Perez (Eds.), Computer games and adult learning (pp. 205-227). Amsterdam, The Netherlands: Elsevier.

Deubel, H., \& Schneider, W. X. (1996). Saccade target selection and object recognition: Evidence for a common attentional mechanism. Vision Research, 36(12), 1827-1837.

Donovan, T., \& Litchfield, D. (2013). Looking for cancer: Expertise related differences in searching and decision making. Applied Cognitive Psychology, 27(1), 43-49.

Druker, M., \& Anderson, B. (2010). Spatial probability aids visual stimulus discrimination. Frontiers in Human Neuroscience, 4(63). doi:10.3389/fnhum.2010.00063

Dye, M. W. G., Green, C. S., \& Bavelier, D. (2009). Increasing speed of processing with action video games. Current Directions in Psychological Science, 18(6), 321-326.

Eckstein, M. P., Drescher, B. A., \& Shimozaki, S. S. (2006). Attentional cues in real scenes, saccadic targeting, and Bayesian priors. Psychological Science, 17(11), 973-980.

Ehinger, K. A., Hidalgo-Sotelo, B., Torralba, A., \& Oliva, A. (2009). Modelling search for people in 900 scenes: A combined source model of eye guidance. Visual Cognition, 17(6/7), 945-978.

Feng, J., Spence, I., \& Pratt, J. (2007). Playing an action video game reduces gender differences in spatial cognition. Psychological Science, 18(10), 850-855.

Franceschini, S., Gori, S., Ruffino, M., Viola, S., Molteni, M., \& Facoetti, A. (2013). Action video games make dyslexic children read better. Current Biology, 23(6), 462-466.

Gaspar, J. G., Neider, M. B., Crowell, J. A., Lutz, A., Kaczmarski, H., \& Kramer, A. F. (2014). Are gamers better crossers? An examination of action video game experience and dual task effects in a simulated street crossing task. Human Factors: The Journal of the Human Factors and Ergonomics Society, 56(3), 443-452.

Green, C. S., \& Bavelier, D. (2003). Action video game modifies visual selective attention. Nature, 423(6939), 534-537.
Green, C. S., \& Bavelier, D. (2006). Effect of action video games on the spatial distribution of visuospatial attention. Journal of Experimental Psychology: Human Perception and Performance, 32(6), 1465-1478.

Green, C. S., Pouget, A., \& Bavelier, D. (2010). Improved probabilistic inference as a general learning mechanism with action video games. Current Biology, 20(17), 1573-1579.

Green, C. S., \& Seitz, A. R. (2015). The impacts of video games on cognition (and how the government can guide the industry). Policy Insights from the Behavioral and Brain Sciences, 2(1), 101-110.

Hayhoe, M., \& Ballard, D. (2005). Eye movements in natural behavior. Trends in Cognitive Sciences, 9(4), 188-194.

Hoffman, J. E., \& Subramaniam, B. (1995). The role of visual attention in saccadic eye movements. Perception \& Psychophysics, 57(6), 787 795.

Hubert-Wallander, B., Green, C. S., Sugarman, M., \& Bavelier, D. (2011). Changes in search rate but not in the dynamics of exogenous attention in action videogame players. Attention, Perception, \& Psychophysics, 73(8), 2399-2412.

Itti, L., \& Koch, C. (2000). A saliency-based search mechanism for overt and covert shifts of visual attention. Vision Research, 40(10), 14891506.

Jones, J. L., \& Kaschak, M. P. (2012). Global statistical learning in a visual search task. Journal of Experimental Psychology: Human Perception and Performance, 38(1), 152-160.

Kanan, C., Tong, M. H., Zhang, L., \& Cottrell, G. W. (2009). SUN: Topdown saliency using natural statistics. Visual Cognition, 17(6/7), 979-1003.

Kingstone, A., Smilek, D., \& Eastwood, J. D. (2008). Cognitive ethology: A new approach for studying human cognition. British Journal of Psychology, 99(3), 317-340.

Kowler, E., Anderson, E., Dosher, B., \& Blaser, E. (1995). The role of attention in the programming of saccades. Vision Research, 35(13), 1897-1916.

Kristjánsson, A. (2013). The case for causal influences of action videogame play upon vision and attention. Attention, Perception \& Psychophysics, 75(4), 667-672.

Land, M. F. (2004). Eye movements in daily life. Visual Neuroscience, 2, $1357-1368$.

Land, M. F., \& Lee, D. N. (1994). Where do we look when we steer. Nature, 369(6483), 742-744.

Land, M. F., \& McLeod, P. (2000). From eye movements to actions: How batsmen hit the ball. Nature Neuroscience, 3(12), 1340 1345.

Li, R., Polat, U., Makous, W., \& Bavelier, D. (2009). Enhancing the contrast sensitivity function through action video game training. Nature Neuroscience, 12(5), 549-551.

Mack, D. J., \& Ilg, U. J. (2014). The effects of video game play on the characteristics of saccadic eye movements. Vision Research, 102, 26-32.

Mackworth, N. H., \& Morandi, A. J. (1967). The gaze selects informative details within pictures. Perception \& Psychophysics, 2(11), 547552.

Oliva, A., Torralba, A., Castelhano, M. S., \& Henderson, J. M. (2003). Top-down control of visual attention in object detection. Paper presented at the International Conference on Image Processing, Barcelona.

Rothkopf, C. A., Ballard, D. H., \& Hayhoe, M. M. (2007). Task and context determine where you look. Journal of Vision, 7(14), 16.

Stainer, M. J., Anderson, A. J., \& Denniss, J. (2015). Examination strategies of experienced and novice clinicians viewing the retina. Ophthalmic and Physiological Optics, 35(4), 424-432.

Torralba, A., Oliva, A., Castelhano, M. S., \& Henderson, J. M. (2006). Contextual guidance of eye movements and attention in real-world 
scenes: The role of global features in object search. Psychological Review, 113(4), 766-786.

West, G. L., Al-Aidroos, N., \& Pratt, J. (2013). Action video game experience affects oculomotor performance. Acta Psychologica, 142(1), $38-42$.

West, G. L., Stevens, S. A., Pun, C., \& Pratt, J. (2008). Visuospatial experience modulates attentional capture: Evidence from action video game players. Journal of Vision, 8(16), 13-13.
Wu, S., \& Spence, I. (2013). Playing shooter and driving videogames improves top-down guidance in visual search. Attention, Perception, \& Psychophysics, 75(4), 673-686.

Yarbus, A. L. (1967). Eye movements and vision. New York, NY: Plenum Press.

Zelinsky, G. J., \& Schmidt, J. (2009). An effect of referential scene constraint on search implies scene segmentation. Visual Cognition, 17(6/7), 1004-1028. 